\title{
THE BEST SOBOLEV TRACE CONSTANT IN PERIODIC MEDIA FOR CRITICAL AND SUBCRITICAL EXPONENTS
}

\author{
JULIÁN FERNÁNDEZ BONDER \\ Departamento de Matemática, FCEyN, Universidad de Buenos Aires, \\ Pabellon I, Ciudad Universitaria (1428), Buenos Aires, Argentina \\ e-mail: jfbonder@dm.uba.ar,web page:http://mate.dm.uba.ar/ jfbonder \\ RAFAEL ORIVE \\ Departamento de Matemáticas, Universidad Autonoma de Madrid, \\ Crta. Colmenar Viejo km. 15, 28049 Madrid, Spain \\ e-mail:rafael.orive@uam.es,web page: http://www.uam.es/rafael.orive \\ and JULIO D. ROSSI \\ Departamento de Matemática, FCEyN, Universidad de Buenos Aires, \\ Pabellon I, Ciudad Universitaria (1428), Buenos Aires, Argentina \\ e-mail: jrossi@dm.uba.ar,web page: http://mate.dm.uba.ar/ jrossi
}

(Received 17 December 2007; accepted 5 February 2009)

\begin{abstract}
In this paper we study homogenisation problems for Sobolev trace embedding $H^{1}(\Omega) \hookrightarrow L^{q}(\partial \Omega)$ in a bounded smooth domain. When $q=2$ this leads to a Steklov-like eigenvalue problem. We deal with the best constant of the Sobolev trace embedding in rapidly oscillating periodic media, and we consider $H^{1}$ and $L^{q}$ spaces with weights that are periodic in space. We find that extremals for these embeddings converge to a solution of a homogenised limit problem, and the best trace constant converges to a homogenised best trace constant. Our results are in fact more general; we can also consider general operators of the form $a^{\varepsilon}(x, \nabla u)$ with non-linear Neumann boundary conditions. In particular, we can deal with the embedding $W^{1, p}(\Omega) \hookrightarrow$ $L^{q}(\partial \Omega)$.
\end{abstract}

2000 Mathematics Subject Classification. 35B27, 35J65, 46E35.

1. Introduction. Sobolev inequalities have been studied by many authors and are by now a classical subject. They at least go back to [3] (for more references see [10]). Relevant for the study of boundary value problems for differential operators is the Sobolev trace inequality that has been intensively studied (see for example $[\mathbf{1 1}, \mathbf{1 2}, 14$ 16]. Given a bounded smooth domain $\Omega \subset \mathbb{R}^{N}$, we deal with the best constant of the Sobolev trace embedding $H^{1}(\Omega) \hookrightarrow L^{q}(\partial \Omega)$. When $q=2$ this leads to an eigenvalue problem of the Steklov type.

Our main goal here is to consider the Sobolev trace inequality for $H^{1}$ and $L^{q}$ spaces with weights that oscillate periodically. We find that extremals for these embeddings converge as the oscillations go to infinity to a solution of a homogenised limit problem, and the best trace constant converges to a homogenised best trace constant. 
Let us consider the following coefficients:

$$
\begin{aligned}
& \left\{\begin{array}{l}
a_{i j} \in L_{\#}^{\infty}(\mathbb{T}), \text { where } \mathbb{T}=[0,1]^{N}, \text { i.e. each } a_{i j} \text { is a } \mathbb{T} \text {-periodic } \\
\text { bounded measurable function defined on } \mathbb{R}^{N}, \\
\exists \alpha, \beta>0 \text { such that } \alpha|\eta|^{2} \leq a_{i j}(x) \eta_{i} \eta_{j} \leq \beta|\eta|^{2} \forall \eta \in \mathbb{R}^{N} \text {, a.e. } x \in \mathbb{T}, \\
a_{i j}=a_{j i} \quad \forall i, j=1, \ldots, N,
\end{array}\right. \\
& \qquad\left\{\begin{array}{l}
a_{0} \in L_{\#}^{\infty}(\mathbb{T}) \text {, i.e. } a_{0} \text { is } \mathbb{T} \text {-periodic, and } \\
\exists a_{-}, a_{+} \in \mathbb{R}_{+}, \text {such that } 0<a_{-} \leq a_{0}(x) \leq a_{+}, \text {a.e. } x \in \mathbb{T}
\end{array}\right.
\end{aligned}
$$

and

$$
\left\{\begin{array}{l}
b \in L_{\#}^{\infty}(\mathbb{T}), \text { i.e. } b \text { is } \mathbb{T} \text {-periodic, and } \\
\exists b_{-}, b_{+} \in \mathbb{R}_{+}, \text {such that } 0<b_{-} \leq b(x) \leq b_{+}, \text {a.e. } x \in \mathbb{T} .
\end{array}\right.
$$

Associated with these coefficients and a parameter $\varepsilon>0$, we consider for every critical or subcritical exponent, $1 \leq q \leq 2_{*}:=2(N-1) /(N-2)$, the Sobolev trace inequality

$$
S(\varepsilon) \int_{\partial \Omega} b^{\varepsilon}|v|^{q} d S \leq \int_{\Omega}\left(a_{i j}^{\varepsilon} \frac{\partial v}{\partial x_{j}} \frac{\partial v}{\partial x_{i}}+a_{0}^{\varepsilon} v^{2}\right) d x,
$$

valid for all $v \in H^{1}(\Omega)$. Here $a_{i j}^{\varepsilon}(x):=a_{i j}(x / \varepsilon), a_{0}^{\varepsilon}(x):=a_{0}(x / \varepsilon)$ and $b^{\varepsilon}(x):=b(x / \varepsilon)$.

The best Sobolev trace constant is the largest $S(\varepsilon)$ such that the above inequality holds, that is

$$
S(\varepsilon):=\inf _{v \in H^{1}(\Omega) \backslash H_{0}^{1}(\Omega)} \frac{\int_{\Omega}\left(a_{i j}^{\varepsilon} \frac{\partial v}{\partial x_{j}} \frac{\partial v}{\partial x_{i}}+a_{0}^{\varepsilon} v^{2}\right) d x}{\left(\int_{\partial \Omega} b^{\varepsilon}|v|^{q} d S\right)^{2 / q}} .
$$

For subcritical exponents, $1 \leq q<2_{*}$, the embedding $H^{1}(\Omega) \hookrightarrow L^{q}(\partial \Omega)$ is compact; so we have existence of extremals, i.e. functions in which the infimum is attained. These extremals are strictly positive in $\Omega$ (see [14]) and smooth up to the boundary (see [6]). When one normalise the extremals with

$$
\int_{\partial \Omega} b^{\varepsilon}\left|u_{\varepsilon}\right|^{q} d S=1
$$

it follows that they are weak solutions of the following problem:

$$
\begin{cases}\frac{\partial}{\partial x_{i}}\left(a_{i j}^{\varepsilon} \frac{\partial u_{\varepsilon}}{\partial x_{j}}\right)=a_{0}^{\varepsilon} u_{\varepsilon} & \text { in } \Omega, \\ \frac{\partial u_{\varepsilon}}{\partial \nu^{\varepsilon}}:=a_{i j}^{\varepsilon} \frac{\partial u_{\varepsilon}}{\partial x_{j}} v_{i}=S(\varepsilon) b^{\varepsilon}\left|u_{\varepsilon}\right|^{q-2} u_{\varepsilon} & \text { on } \partial \Omega,\end{cases}
$$


where $v$ is the unit outward normal vector. Of special importance is the case $q=2$. In this case, (1.6) is an eigenvalue problem of Steklov type (see [20]). In the rest of the paper we will assume that the extremals are normalised according to (1.5).

Our first result is the following.

TheORem 1. Let $1 \leq q<2 *$. Assume that $\Omega$ is a generic domain; that is assume that the boundary of $\Omega, \partial \Omega$, does not contain flat pieces or that it contains finitely many flat pieces with conormal and not proportional to any $m \in \mathbb{Z}^{N}$. Then, the function $S(\varepsilon)$ converges as $\varepsilon \rightarrow 0$ to $S^{*}$, the best Sobolev trace constant of the homogenised problem that is defined by

$$
S^{*}=\inf _{v \in H^{1}(\Omega) \backslash H_{0}^{1}(\Omega)} \frac{\int_{\Omega}\left(a_{\ddot{j}}^{*} \frac{\partial v}{\partial x_{j}} \frac{\partial v}{\partial x_{i}}+a_{0}^{*} v^{2}\right) d x}{\left(\int_{\partial \Omega} b^{*}|v|^{q} d S\right)^{2 / q}},
$$

where the homogenised coefficients are defined by $a_{0}^{*}$ and $b^{*}$, which are the mean value of $a_{0}$ and $b$ respectively, i.e.

$$
a_{0}^{*}:=\int_{\mathbb{T}} a_{0}(y) d y, \quad b^{*}:=\int_{\mathbb{T}} b(y) d y .
$$

The coefficients $a_{i j}^{*}$ are given by

$$
a_{i j}^{*}:=\int_{\mathbb{T}}\left(a_{i j}-\frac{\partial a_{i \ell}}{\partial y_{\ell}} \chi_{j}\right) d y,
$$

where for any $k=1, \ldots, d, \chi_{k}$ is the unique solution of the cell problem

$$
\left\{\begin{array}{l}
-\frac{\partial}{\partial y_{i}}\left(a_{i j} \frac{\partial \chi_{k}}{\partial y_{j}}\right)=\frac{\partial a_{k \ell}}{\partial y_{\ell}} \quad \text { in } \mathbb{T} \\
\chi_{k} \in H_{\#}^{1}(\mathbb{T}), \quad m\left(\chi_{k}\right)=0 .
\end{array}\right.
$$

Moreover, as $\varepsilon \rightarrow 0$ the sequence of extremals $\left\{u_{\varepsilon}\right\}$ of (1.4) converges (along subsequences) weakly in $H^{1}(\Omega)$ to a limit $u^{*}$ that is an extremal of the homogenised problem (1.7), and so, it verifies

$$
\begin{cases}\frac{\partial}{\partial x_{i}}\left(a_{i j}^{*} \frac{\partial u^{*}}{\partial x_{j}}\right)=a_{0}^{*} u^{*} & \text { in } \Omega, \\ \frac{\partial u^{*}}{\partial \nu^{*}}:=a_{i j}^{*} \frac{\partial v^{*}}{\partial x_{j}} v_{i}=S^{*} b^{*}\left|u^{*}\right|^{q-2} u^{*} & \text { on } \partial \Omega .\end{cases}
$$

REMARK 1.1. The homogenised coefficients are related to the original coefficients by the usual homogenisation rules (see [5]). Concerning boundary terms, in [17], it is proved that for generic domains there exists a limit. However for non-generic domains there exist different limits for different sequences of $\varepsilon \rightarrow 0$. In Theorem 1 we consider the generic case; that is we impose that the boundary of $\Omega$ does not contain flat pieces or that it contains finitely many flat pieces with conormal and not proportional to any $m \in \mathbb{Z}^{N}$. 
REMARK 1.2. This result can be generalised using $H$-convergence. If we have a sequence of coefficients $\left(a_{i j}^{\varepsilon}\right)$ that converges to $\left(a_{i j}^{*}\right)$ in the sense of $H$-convergence (see [18]), then the corresponding extremals $u_{\varepsilon}$ converge weakly in $H^{1}(\Omega)$ to an extremal of the limit problem. To see this fact we only have to observe that using $H$-convergence, we can pass to the limit in the weak form of equation (1.6).

This result can also be seen from the $\Gamma$-convergence of functionals' point of view. The functionals describe the stored energy of the portion of the $\varepsilon$-periodic composite material occupying a region $\Omega$ of $\mathbb{R}^{N}$. The $\Gamma$-convergence provides the behaviour of the extremals and the shape of the limit of the functionals (see [9] for an extensive study of this method).

Our second result deals with the critical exponent, $q=2_{*}$. In this case, under a geometric assumption on the domain, we have a similar result.

Theorem 2. Assume that $\Omega$ is a generic domain (see Theorem 1) and that

$$
\frac{\alpha(N-2)|B(0,1)|^{1 /(N-1)}}{2\left(b_{+}\right)^{2 / 2_{*}}}>\frac{|\Omega| a_{+}}{|\partial \Omega| b_{-}},
$$

where the constants $\alpha, a_{+}$and $b_{ \pm}$are given in (1.1)-(1.3).

Then, the function $S(\varepsilon)$ converges as $\varepsilon \rightarrow 0$ to $S^{*}$, the best Sobolev trace constant of the homogenised problem that is defined by (1.7) Moreover, as $\varepsilon \rightarrow 0$ the sequence of extremals $\left\{u_{\varepsilon}\right\}$ of (1.4) converges (along subsequences) weakly in $H^{1}(\Omega)$ to a limit $u^{*}$ that is an extremal of the homogenised problem (1.7) (and, so, a solution of (1.11)).

REMARK 1.3. In the proof of Theorem 2, what is actually used is that there exists $\delta>0$ (independent of $\varepsilon$ ) such that $S(\varepsilon)$ satisfies

$$
\frac{\alpha(N-2)|B(0,1)|^{1 /(N-1)}}{2\left(b_{+}\right)^{2 / 2_{*}}}-\delta>S(\varepsilon) .
$$

This condition is implied by (1.12), taking $u \equiv 1$ as a test function in (1.4).

Arguing as in [15], one can check that hypothesis (1.13) implies the existence of an extremal $u_{\varepsilon}$ for (1.4).

Our results are in fact more general. For the sake of clarity we choose to present first the linear case with periodic coefficients in full detail. However, using ideas from [4], we can deal with more general (non-linear) operators.

Let $a^{\varepsilon}(x, \xi)$ and $b^{\varepsilon}(x, u)$, with $x \in \Omega, \xi \in \mathbb{R}^{N}$ and $u \in \mathbb{R}$, be general non-linear functions verifying convenient hypotheses (see Section 5 ). We consider

$$
\lambda_{1}=\inf _{v \in W^{1, p}(\Omega) \backslash W_{0}^{1, p}(\Omega)} \frac{\int_{\Omega} a(x, \nabla v) \cdot \nabla v+b(x, v) v d x}{\int_{\partial \Omega}|v|^{q} d S} .
$$

THEOREM 3. Assume that $a^{\varepsilon}$ and $b^{\varepsilon}$ satisfy hypotheses (A1)-(A4), (B1)-(B3) in Section 5 and that there exist two limit functions $a_{\mathrm{hom}}: \Omega \times \mathbb{R}^{N} \rightarrow \mathbb{R}^{N}$ and $b_{\mathrm{hom}}: \Omega \times$ $\mathbb{R} \rightarrow \mathbb{R}$ that satisfy the same hypotheses. 
Also assume that the operators $\mathcal{A}^{\varepsilon} G$-converge to the operator $\mathcal{A}_{\mathrm{hom}}$ associated with these functions. Let $\lambda_{1}^{\varepsilon}$ and $\lambda_{1}^{\text {hom }}$ be as in (1.14) with $a, b$ replaced by $a^{\varepsilon}, b^{\varepsilon}$ and $a_{\mathrm{hom}}, b_{\mathrm{hom}}$ respectively.

1. If $1 \leq q<p_{*}:=p(N-1) /(N-p)$, then, $\lambda_{1}^{\varepsilon} \rightarrow \lambda_{1}^{\text {hom }}$ as $\varepsilon \rightarrow 0$.

Moreover, the extremals $\left\{u_{\varepsilon}\right\}$ converge (along subsequences) weakly in $W^{1, p}(\Omega)$ to a limit $u^{*}$ that is an extremal of the homogenised problem.

2. For the critical case, $q=p_{*}$, assume that $\Omega$ verifies

$$
\frac{|\Omega|}{|\partial \Omega|^{* / p}}<\frac{c}{K(N, p)}
$$

where $K(N, p)$ is the best Sobolev trace constant in a half-space,

$$
K(N, p)=\inf _{\nabla v \in L^{p}\left(\mathbb{R}_{+}^{N}\right), w \in L^{p^{*}}\left(\partial \mathbb{R}_{+}^{N}\right)} \frac{\int_{\mathbb{R}_{+}^{N}}|\nabla v|^{p} d x}{\left(\int_{\partial \mathbb{R}_{+}^{N}}|v|^{p^{*}} d S\right)^{p / p^{*}},}
$$

and $c$ depend on the family of coefficients. Then, the conclusions of the previous item hold true.

To end this introduction, let us mention that homogenisation results for the Sobolev trace constant in domains with holes for critical and subcritical exponents have been recently considered in [13] in the spirit of [8].

The rest of the paper is organised as follows: in Section 2 we recall some preliminary results that are needed in the proof of the main theorems; in Section 3 we deal with the subcritical case (Theorem 1) and in Section 4 with the critical case (Theorem 2); and, finally, in Section 5 we prove the extension for the non-linear case (Theorem 3).

2. Preliminaries. In this subsection we present some results and techniques in homogenisation of periodic media. We briefly recall the notion of two-scale convergence (see [2], [19]).

Proposition 2.1. Let $\Omega \subseteq \mathbb{R}^{N}$ and $w_{\varepsilon}$ be a bounded sequence in $L^{2}(\Omega)$. There exist a subsequence, still denoted by $\varepsilon$, and a limit $w(x, y) \in L^{2}\left(\Omega ; L_{\#}^{2}(\mathbb{T})\right)$ such that $w_{\varepsilon}$ two-scale converges to $w$ in the sense that

$$
\lim _{\varepsilon \rightarrow 0} \int_{\Omega} w_{\varepsilon}(x) \phi(x, x / \varepsilon) d x=\int_{\Omega} \int_{\mathbb{T}} w(x, y) \phi(x, y) d x d y
$$

for every function $\phi(x, y) \in L^{2}\left(\Omega ; C_{\#}(\mathbb{T})\right)$. The two-scale convergence is denoted by $w_{\varepsilon} \rightarrow$ $w$ in $2 s$. Furthermore, if $\left\{w_{\varepsilon}\right\}$ is a bounded sequence that converges weakly to a limit $w$ in $H^{1}(\Omega)$, then, $w_{\varepsilon}$ two-scale converges to $w$, and there exists a function $w_{1}(x, y) \in$ $L^{2}\left(\Omega ; H_{\#}^{1}(\mathbb{T})\right)$ such that up to a subsequence, we have the following two-scale convergence:

$$
\nabla w_{\varepsilon}(x) \rightarrow \nabla_{x} w(x)+\nabla_{y} w_{1}(x, y) \quad \text { in two-scale. }
$$

This two-scale convergence result is a powerful tool to deal with our problem, the study of the limit as $\varepsilon \rightarrow 0$ in (1.4). 
Another important tool is the weak star convergence in $L^{\infty}(\Omega)$. In general, if $g_{\varepsilon}, g \in L^{\infty}(\Omega)$, we say that $g_{\varepsilon}$ converges to $g$ weak star in $L^{\infty}(\Omega)$, denoted by $g_{\varepsilon} \stackrel{*}{\rightarrow} g$ in $L^{\infty}(\Omega)$, if

$$
\int_{\Omega} g_{\varepsilon} \phi d x \rightarrow \int_{\Omega} g \phi d x, \quad \forall \phi \in L^{1}(\Omega) .
$$

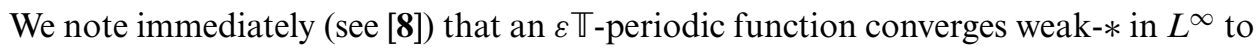
its mean value. Thus

$$
a_{0}^{\varepsilon} \stackrel{*}{\rightarrow} a_{0}^{*} \quad \text { in } L^{\infty}(\Omega) .
$$

Moreover, if $\Omega$ is a generic domain, i.e. $\partial \Omega$ does not contain flat pieces or that it contains finitely many flat pieces with conormal and not proportional to any $m \in \mathbb{Z}^{N}$, we have that

$$
b^{\varepsilon} \stackrel{*}{\rightarrow} b^{*} \quad \text { in } L^{\infty}(\partial \Omega),
$$

where $b^{*}$ is given by (1.8) (see Remark 1.1).

3. Subcritical case. In this section we assume that $q$ is subcritical, that is $1 \leq q<$ $2_{*}$; so the immersion $H^{1}(\Omega) \hookrightarrow L^{q}(\partial \Omega)$ is compact.

Proof of Theorem 1. First, let us prove that the best constants $S(\varepsilon)$ and the extremals $u_{\varepsilon}$ are bounded in $H^{1}$ independent of $\varepsilon$. Indeed, by the definition of $S(\varepsilon)$ in (1.4) and our assumptions on coefficients (1.1) and (1.2), there exist two constants $0<c<C$ such that

$$
c \lambda_{0} \leq S(\varepsilon) \leq C \lambda_{0}
$$

with $\lambda_{0}$ defined by

$$
\lambda_{0}=\inf _{v \in H^{1}(\Omega) \backslash H_{0}^{1}(\Omega)} \frac{\int_{\Omega}|\nabla v|^{2}+v^{2} d x}{\left(\int_{\partial \Omega}|v|^{q} d S\right)^{2 / q}} .
$$

Now, we show that the extremals $u_{\varepsilon}$, the weak solutions of (1.6), are bounded in $H^{1}$-norm independent of $\varepsilon$. To prove this fact recall that we have normalised the extremals by (1.5). By our assumptions on coefficients (1.1) and (1.2), we have

$$
S(\varepsilon)=\int_{\Omega}\left(a_{i j}^{\varepsilon} \frac{\partial u_{\varepsilon}}{\partial x_{j}} \frac{\partial u_{\varepsilon}}{\partial x_{i}}+a_{0}^{\varepsilon}\left|u_{\varepsilon}\right|^{2}\right) d x \geq \int_{\Omega}\left(\alpha\left|\nabla u_{\varepsilon}\right|^{2}+a_{-}\left|u_{\varepsilon}\right|^{2}\right) d x .
$$

By (3.1), we obtain that $u_{\varepsilon}$ is bounded in $H^{1}$ independent of $\varepsilon$. Hence there exists a subsequence (which we still call $u_{\varepsilon}$ ) and a function $u_{0} \in H^{1}(\Omega)$ such that $u_{\varepsilon} \rightarrow u_{0}$ weakly in $H^{1}(\Omega)$ and $u_{\varepsilon} \rightarrow u_{0}$ strongly in $L^{q}(\partial \Omega)$ for $1 \leq q<2_{*}$. By the above-mentioned convergence and (2.1) we have that

$$
\int_{\partial \Omega} b^{*}\left|u_{0}\right|^{q} d S=1
$$


Moreover, using Proposition 2.1, we obtain that $u_{\varepsilon} \rightarrow u_{0}$ in two-scale, and there exists $u_{1}$ such that

$$
\nabla u_{\varepsilon} \rightarrow \nabla_{x} u_{0}(x)+\nabla_{y} u_{1}(x, y), \quad \text { in two-scale. }
$$

We use $\phi(x)+\varepsilon \phi_{1}(x, x / \varepsilon)$ with $\phi \in H^{1}(\Omega)$ and $\phi_{1} \in H^{1}\left(\Omega ; C_{\#}(\mathbb{T})\right)$ as a test function in the weak form of (1.6). As $S(\varepsilon)$ is bounded, we can assume that $S(\varepsilon) \rightarrow S_{0}$, for an appropriate subsequence. Then we pass to the limit the weak formulation, and by the two-scale convergence, we get

$$
\begin{aligned}
& \int_{\Omega} \int_{\mathbb{T}} a_{i j}(y)\left(\frac{\partial u_{0}}{\partial x_{j}}(x)+\frac{\partial u_{1}}{\partial y_{j}}(x, y)\right)\left(\frac{\partial \phi}{\partial x_{i}}(x)+\frac{\partial \phi_{1}}{\partial y_{i}}(x, y)\right) d y d x \\
& +\int_{\Omega} \int_{\mathbb{T}} a_{0}(y) u_{0}(x) \phi(x) d x d y=S_{0} \int_{\partial \Omega} \int_{\mathbb{T}} b(y)\left|u_{0}\right|^{q-2} u_{0} \phi(x) d S d y .
\end{aligned}
$$

Integrating by parts we obtain that $\left(u_{0}, u_{1}\right)$ is the weak solution of the system

$$
\begin{aligned}
& \frac{\partial}{\partial x_{i}}\left(\int_{\mathbb{T}} a_{i j}(y)\left(\frac{\partial u_{0}}{\partial x_{j}}(x)+\frac{\partial u_{1}}{\partial y_{j}}(x, y)\right) d y\right)=a_{0}^{*} u_{0}(x) \quad \text { in } \Omega, \\
& v_{i} \frac{\partial}{\partial x_{i}}\left(\int_{\mathbb{T}} a_{i j}(y)\left(\frac{\partial u^{*}}{\partial x_{j}}(x)+\frac{\partial u_{1}}{\partial y_{j}}(x, y)\right) d y\right)=S_{0} b^{*}\left|u_{0}\right|^{q-2} u_{0}(x) \quad \text { on } \partial \Omega, \\
& \frac{\partial}{\partial y_{i}}\left(a_{i j}(y)\left(\frac{\partial u_{0}}{\partial x_{i}}(x)+\frac{\partial u_{1}}{\partial y_{i}}(x, y)\right)\right)=0 \quad \text { in } \Omega \times \mathbb{T},
\end{aligned}
$$

with $a_{0}^{*}$ and $b^{*}$ defined in (1.8). Considering

$$
u_{1}(x, y)=\sum_{i=1}^{N} \frac{\partial u_{0}}{\partial x_{i}}(x) \chi_{i}(y),
$$

we note that $u_{1}$ satisfies (3.5) for any $u_{0}$, since $\chi_{1}$ is the solution of (1.10). Moreover, with this function $u_{1}$ in (3.3) and (3.4), we obtain that $u_{0}$ is a solution of

$$
\begin{cases}\frac{\partial}{\partial x_{i}}\left(a_{\ddot{j}}^{*} \frac{\partial u_{0}}{\partial x_{j}}\right)=a_{0}^{*} u_{0} & \text { in } \Omega, \\ \frac{\partial u_{0}}{\partial \nu^{*}}=S_{0} b^{*}\left|u_{0}\right|^{q-2} u_{0} & \text { on } \partial \Omega,\end{cases}
$$

where the coefficients $a_{i j}^{*}$ are given by (1.9) and the derivative normal $\partial / \partial \nu^{*}$ is defined in (1.11). Now, since $S_{0}$ satisfies (3.6), we get $S_{0} \geq S^{*}$ with $S^{*}$ defined in (1.7). To conclude the proof of Theorem 1 we need to show that $S_{0}=S^{*}$. In fact, let $u^{*}$ be an extremal of (1.7) and consider

$$
v_{\varepsilon}=u^{*}+\varepsilon \chi_{k}^{\varepsilon} \frac{\partial u^{*}}{\partial x_{k}}
$$

as a test function in (1.4), where $\chi_{k}^{\varepsilon}(x)=\chi_{k}(x / \varepsilon)$. From the maximum principle and Hopf's lemma we get that $u^{*}$ is strictly positive in $\bar{\Omega}$. Therefore the regularity results of [6] are applicable, and we obtain that $u^{*} \in C^{\infty}(\bar{\Omega})$. Thus, since the functions $\chi_{k} \in W^{1, \infty}$ (this is a consequence of the hypotheses on the coefficients), we have immediately 
$v_{\varepsilon} \rightarrow u^{*}$ weakly in $H^{1}(\Omega)$ and $v_{\varepsilon} \rightarrow u^{*}$ strongly in $L^{q}(\partial \Omega)$ for $1 \leq q<2^{*}$. Now, we obtain

$$
\begin{aligned}
\int_{\Omega}\left(a_{i j}^{\varepsilon} \frac{\partial v_{\varepsilon}}{\partial x_{j}} \frac{\partial v_{\varepsilon}}{\partial x_{i}}+a_{0}^{\varepsilon} v_{\varepsilon}^{2}\right) d x= & \int_{\Omega}\left(a_{i j}^{\varepsilon}+a_{i k}^{\varepsilon} \frac{\partial \chi_{j}^{\varepsilon}}{\partial y_{k}}\right) \frac{\partial u^{*}}{\partial x_{j}} \frac{\partial u^{*}}{\partial x_{i}} d x \\
& +\int_{\Omega}\left(a_{i j}^{\varepsilon} \frac{\partial \chi_{k}^{\varepsilon}}{\partial y_{j}} \frac{\partial \chi_{\ell}^{\varepsilon}}{\partial y_{i}}+a_{i k}^{\varepsilon} \frac{\partial \chi_{\ell}^{\varepsilon}}{\partial y_{i}}\right) \frac{\partial u^{*}}{\partial x_{k}} \frac{\partial u^{*}}{\partial x_{\ell}} d x \\
& +\int_{\Omega} a_{0}^{\varepsilon}\left(u^{*}\right)^{2} d x+O(\varepsilon) .
\end{aligned}
$$

Passing to the limit, using that $\chi_{k}$ is a solution of (1.10) and by the weak-* convergence in $L^{\infty}$, we get

$$
\lim _{\varepsilon \rightarrow 0} \int_{\Omega}\left(a_{i j}^{\varepsilon} \frac{\partial v_{\varepsilon}}{\partial x_{j}} \frac{\partial v_{\varepsilon}}{\partial x_{i}}+a_{0}^{\varepsilon} v_{\varepsilon}^{2}\right) d x=\int_{\Omega}\left(a_{i j}^{*} \frac{\partial u^{*}}{\partial x_{j}} \frac{\partial u^{*}}{\partial x_{i}}+a_{0}^{*}\left(u^{*}\right)^{2}\right) d x,
$$

where $a_{0}^{*}$ and $a_{i j}^{*}$ are defined by (1.8) and (1.9), respectively. Moreover, again by the weak-* convergence in $L^{\infty}$, we have

$$
\int_{\partial \Omega} b^{\varepsilon}\left|v_{\varepsilon}\right|^{q} \rightarrow \int_{\partial \Omega} b^{*}\left|u^{*}\right|^{q}
$$

Therefore, passing to the limit in (1.4) with test function $v_{\varepsilon}$, we prove $S_{0} \leq S^{*}$, and we conclude the proof of Theorem 1 .

REMARK 3.1. Results on correctors of the extremals are easily obtained with the two-scale convergence method. Considering the solutions of the cell problem (1.10), the corrector term is defined by

$$
u_{1}^{\varepsilon}(x)=\chi_{k}(x / \varepsilon) \frac{\partial u^{*}}{\partial x_{k}}(x)
$$

where $u^{*}$ is an extremal of the homogenised problem (1.11). Hence, by Proposition 2.1 and following the same lines as [2], $\left(u^{\varepsilon}-u^{*}-\varepsilon u_{1}^{\varepsilon}\right)$ converges strongly to zero in $H^{1}(\Omega)$.

4. Critical case. In this section we deal with the critical exponent $q=2_{*}=2(\mathrm{~N}-$ $1) /(N-2)$.

Proof of Theorem 2. Recall that as observed in Remark 1.3, hypothesis (1.12) implies the existence of an extremal $u_{\varepsilon}$ for (1.4).

As before, by the definition of $S(\varepsilon)$ in (1.4) and our assumptions on coefficients (1.1) and (1.2), we have (3.1). Hence, the extremals $u_{\varepsilon}$ are bounded in $H^{1}(\Omega)$ and we have, for a subsequence,

$$
\begin{array}{ll}
u_{\varepsilon} \rightarrow u_{0} & \text { weakly in } H^{1}(\Omega), \\
u_{\varepsilon} \rightarrow u_{0} & \text { strongly in } L^{q}(\partial \Omega),
\end{array}
$$


Arguing exactly as in the previous section we obtain that $u_{\varepsilon} \rightarrow u_{0}$ in $2 s$ and, moreover, that $u_{0}$ is a weak solution to

$$
\begin{cases}\frac{\partial}{\partial x_{i}}\left(a_{i j}^{*} \frac{\partial u_{0}}{\partial x_{j}}\right)=a_{0}^{*} u_{0} & \text { in } \Omega, \\ \frac{\partial u_{0}}{\partial v^{*}}=S b^{*}\left|u_{0}\right|^{q-2} u_{0} & \text { on } \partial \Omega,\end{cases}
$$

where $S$ is the limit of a subsequence of $S(\varepsilon)$; the coefficients $a_{i j}^{*}$ are given by (1.9); and the derivative normal $\partial / \partial \nu^{*}$ is defined in (1.11).

Let us prove that $u_{0} \neq 0$. To this end we use the following Theorem due to [16].

THEOREM 4. There exists a constant $B>0$ such that

$$
\left(\int_{\partial \Omega} v^{2 *} d S\right)^{2 / 2_{*}} \leq A \int_{\Omega}|\nabla v|^{2} d x+B \int_{\Omega} v^{2} d x
$$

for every $v \in H^{1}(\Omega)$, where

$$
A=\frac{2}{(N-2)|B(0,1)|^{1 /(N-1)}} .
$$

REMARK 4.1. The constant $A$ in Theorem 4 is sharp.

Now, as $u_{\varepsilon} \geq 0$, it follows that $u_{0} \geq 0$ and, by classical regularity theory, $u_{0}$ is smooth up to the boundary. By the strong maximum principle and Hopf's lemma, it follows that either $u_{0}>0$ or $u_{0} \equiv 0$. In order to prove of the result, we have to exclude this last possibility. To this end, we use the argument given in [15] to show that $\left\|u_{0}\right\|_{L^{2}(\Omega)} \neq 0$. In fact, by Theorem 4 , we have that there exists a constant $B$ such that

$$
\left(\int_{\partial \Omega} v^{2_{*}} d \sigma\right)^{2 / 2_{*}} \leq A \int_{\Omega}|\nabla v|^{2} d x+B \int_{\Omega}|v|^{2} d x
$$

for every $v \in H^{1}(\Omega)$. Recall that $u_{\varepsilon}$ are normalised such that (1.5) is satisfied; so, by (1.3),

$$
1=\left(\int_{\partial \Omega} b^{\varepsilon} u_{\varepsilon}^{2_{*}} d \sigma\right)^{2 / 2_{*}} \leq\left(b_{+}\right)^{2 / 2_{*}}\left(A \int_{\Omega}\left|\nabla u_{\varepsilon}\right|^{2} d x+B \int_{\Omega} u_{\varepsilon}^{2} d x\right) .
$$

Hence, for some suitable $\tilde{B}$ we get

$$
\frac{1}{\left(b_{+}\right)^{2 / 2_{*}}} \leq \frac{A}{\alpha}\left(\int_{\Omega} a_{i j}^{\varepsilon} \frac{\partial u_{\varepsilon}}{\partial x_{j}} \frac{\partial u_{\varepsilon}}{\partial x_{i}} d x+\int_{\Omega} a_{0}^{\varepsilon}\left|u_{\varepsilon}\right|^{2} d x\right)+\tilde{B}\left(\int_{\Omega} u_{\varepsilon}^{2} d x\right) .
$$

Therefore,

$$
\frac{1}{\left(b_{+}\right)^{2 / 2_{*}}} \leq \frac{A}{\alpha} S(\varepsilon)+\tilde{B} \int_{\Omega}\left|u_{\varepsilon}\right|^{2} d x .
$$

Passing to the limit $\varepsilon \rightarrow 0$ in (4.2) we arrive to

$$
\frac{1}{\left(b_{+}\right)^{2 / 2_{*}}} \leq \frac{A}{\alpha} S+\tilde{B} \int_{\Omega}\left|u_{0}\right|^{2} d x
$$


therefore, as we have assumed (1.13), which implies

$$
\frac{\alpha}{A\left(b_{+}\right)^{2 / 2_{*}}}>S
$$

we conclude $u_{0} \neq 0$.

Now, multiplying (4.1) by $u_{0}$ and integrating by parts, we obtain

$$
\int_{\Omega} a_{i j}^{*} \frac{\partial u_{0}}{\partial x_{j}} \frac{\partial u_{0}}{\partial x_{i}}+a_{0}^{*} u_{0}^{2} d x=S \int_{\partial \Omega} u_{0}^{2 *} d S .
$$

As $u_{0} \neq 0$ it follows that $S \neq 0$ and $\left\|u_{0}\right\|_{L^{2 *}(\partial \Omega)} \neq 0$. Therefore, we conclude that

$$
S_{0} \leq \frac{\int_{\Omega} a_{i j}^{*} \frac{\partial u_{0}}{\partial x_{j}} \frac{\partial u_{0}}{\partial x_{i}}+a_{0}^{*} u_{0}^{2} d x}{\left(\int_{\partial \Omega} u_{0}^{2_{*}} d S\right)^{2 / 2_{*}}}=S\left(\int_{\partial \Omega} u_{0}^{2_{*}} d S\right)^{1 /(N-1)} \leq S .
$$

Now, arguing exactly as in the end of Section 3, we conclude the desired result.

5. The non-linear case. Finally, in this section we consider the extension of our previous results to a more general class of non-linear operators, including the $p$ Laplacian with oscillating coefficients. The main ideas for these extensions are similar to the ones used before in combination with those of [4]. form

We consider non-linear monotone operators $\mathcal{A}: W^{1, p}(\Omega) \rightarrow\left(W^{1, p}(\Omega)\right)^{*}$ of the

$$
\mathcal{A} u=-\operatorname{div}(a(x, \nabla u))+b(x, u),
$$

whose coefficients $a: \Omega \times \mathbb{R}^{N} \rightarrow \mathbb{R}^{N}$ belong to the class of functions satisfying the following hypotheses:

(A1) $a(\cdot, \cdot)$ is of Carathéodory type.

(A2) Monotonicity: $0 \leq\left(a\left(x, \xi_{1}\right)-a\left(x, \xi_{2}\right)\right) \cdot\left(\xi_{1}-\xi_{2}\right), \quad \forall \xi_{1}$, $\xi_{2}$, a.e. x.

(A3) Uniform ellipticity: $\alpha|\xi|^{p} \leq a(x, \xi) \cdot \xi, \quad \forall \xi$, a.e. $\mathrm{x}$.

(A4) Growth: $|a(x, \xi)| \leq \beta|\xi|^{p-1}, \quad \forall \xi$, a.e. $\mathrm{x}$.

And the function $b: \Omega \times \mathbb{R} \rightarrow \mathbb{R}$ satisfies the following hypotheses:

(B1) $b(\cdot, \cdot)$ is of Carathéodory type.

(B2) Uniform $\alpha|u|^{p} \leq b(x, u) u, \quad \forall u$, a.e. $\mathrm{x}$.

(B3) Growth: $|b(x, u)| \leq \beta|u|^{p-1}, \quad \forall u$, a.e. $\mathrm{x}$.

For $a$ and $b$ satisfying the above hypotheses, we consider the eigenvalue problem

$$
\begin{array}{ll}
\operatorname{div}(a(x, \nabla u))=b(x, u) & \text { in } \Omega, \\
a(x, \nabla u) \cdot v=\lambda|u|^{q-2} u & \text { on } \partial \Omega .
\end{array}
$$

If there exist $\lambda$ and $u$ solutions of (5.1), taking $u$ as a test function in the eigenvalue problem, we note that

$$
\lambda=\frac{\int_{\Omega} a(x, \nabla u) \cdot \nabla u+b(x, u) u d x}{\int_{\partial \Omega}|u|^{q} d S} .
$$


Moreover, the infimum in (1.14) is attained and is called the first eigenvalue $\lambda_{1}$ for the problem (5.1). This fact is indeed by the lower semi-continuity property of the functional associated with $\mathcal{A}$ for the minimising sequence.

Let $\varepsilon>0$ be a small parameter which represents the scale of heterogeneity. We consider a family of functions $a^{\varepsilon}, b^{\varepsilon}$ satisfying the previous hypotheses, for example, $a^{\varepsilon}(x, \xi)=a(x / \varepsilon, \xi)$ and $b^{\varepsilon}(x, u)=b(x / \varepsilon, u)$ which are, in addition, periodic in $x$. Thus, we deal with the minimisation problem

$$
\lambda_{1}^{\varepsilon}=\inf _{v \in W^{1, p}(\Omega) \backslash W_{0}^{1, p}(\Omega)} \frac{\int_{\Omega} a^{\varepsilon}(x, \nabla v) \cdot \nabla v+b^{\varepsilon}(x, v) v d x}{\int_{\partial \Omega}|v|^{q} d S} .
$$

First, assume that $q$ is subcritical. Then, since the embedding $W^{1, p}(\Omega) \hookrightarrow L^{q}(\partial \Omega)$ is compact there exist extremals for (5.3). We normalise the extremals with the condition

$$
\int_{\partial \Omega}\left|u_{\varepsilon}\right|^{q} d S=1 .
$$

The normalised extremals are weak solutions of the problem

$$
\begin{aligned}
\operatorname{div}\left(a^{\varepsilon}\left(x, \nabla u_{\varepsilon}\right)\right)=b^{\varepsilon}\left(x, u_{\varepsilon}\right) u_{\varepsilon} & \text { in } \Omega, \\
a\left(x, \nabla u_{\varepsilon}\right) \cdot v=\lambda_{1}^{\varepsilon}\left|u_{\varepsilon}\right|^{q-2} u_{\varepsilon} & \text { on } \partial \Omega .
\end{aligned}
$$

Since in the statement of Theorem 3 we have assumed the G-convergence of the operators the conclusions concerning the convergence of the first eigenvalue and its associated extremals follow.

Note that this assumption is not restrictive, since if $a^{\varepsilon}$ and $b^{\varepsilon}$ are measurable coefficients which satisfy (A1) $\hat{\mathrm{I}}-(\mathrm{A} 3)$ and (B1)-(B3), then the operators $\mathcal{A}^{\varepsilon}$ G-converge (up to a subsequence) to a maximal monotone operator $\mathcal{A}_{\text {hom }}$ whose coefficients, $a_{\text {hom }}$ and $b_{\text {hom }}$, are measurable and which satisfies (A1)-î-(A3) and (B1)-(B3). We refer to Theorem 4.1 of [7] for this well-known compactness result for the G-convergence on the class of multi-valued functions of the type $a$.

For the critical case $p^{*}=p(N-1) /(N-2)$ we can argue exactly as before in Section 4 , noting that condition (1.15) on the domain and the coefficients involved imply that there are minimisers of (5.3), since some compactness is recovered.

ACKNOWLEDGEMENTS. The first and third authors are supported by CONICET, ANPCyT PICT 05009 and UBA X066. The second author was partially supported by grants S-0505/ESP/0158 of the CAM (Spain) and MTM2005-00715 and MTM200505980 of the MEC (Spain).

\section{REFERENCES}

1. S. L. Adimurthi and S. L. Yadava, Positive solution for Neumann problem with critical non linearity on boundary, Comm. Partial Diff. Eq. 16(11) (1991), 1733-1760.

2. G. Allaire, Homogenization and two-scale convergence, SIAM J. Math. Anal. 23(1992), $1482-1518$.

3. T. Aubin, Équations différentielles non linéaires et le problème de Yamabe concernant la courbure scalaire, J. Math. Pures Appl. 55 (1976), 269-296.

4. L. Baffico, C. Conca and M. Rajesh, Homogenization of a class of nonlinear eigenvalue problems, Proc. R. Soc. Edinb. 136A (2006), 722. 
5. A. Bensoussan, J. L. Lions and G. Papanicolaou, Asymptotic analysis for periodic structures (North-Holland, Amsterdam, 1978).

6. P. Cherrier, Problèmes de Neumann non linéaires sur les variétés Riemanniennes, $J$. Funct. Anal. 57 (1984), 154-206.

7. V. Chiado Piat, G. Dal Maso and A. Defranceschi, G-convergence of monotone operators, Ann. Inst. H. Poincaré 7 (1990), 123-160.

8. D. Cioranescu and F. Murat, A strange term coming from nowhere, in Topics in the mathematical modelling of composite materials (Cherkaev A. and Kohn R., Editors), Progress in Nonlinear Differential Equations and Their Applications, vol. 31 (Birkhäuser, Boston, 1997), 45-93.

9. G. Dal Maso, An introduction to $\Gamma$-convergence, Progress in Nonlinear Differential Equations and Their Applications, vol. 8 (Birkhäuser, Boston, 1993).

10. O. Druet and E. Hebey, The $A B$ program in geometric analysis: Sharp Sobolev inequalities and related problems, Mem. Am. Math. Soc. 160 (2002), 761.

11. J. F. Escobar, Sharp constant in a Sobolev trace inequality, Indiana Math. J. 37(3) (1988), 687-698.

12. J. Fernández Bonder, E. Lami Dozo and J. D. Rossi, Symmetry properties for the extremals of the Sobolev trace embedding, Ann. Inst. H. Poincaré, Anal. Non Linéaire 21(6) (2004), 795-805.

13. J. Fernández Bonder, R. Orive and J. D. Rossi, The best Sobolev trace constant in domains with holes for critical or subcritical exponents, ANZIAM J. 49 (2007), 213-230.

14. J. Fernández Bonder and J. D. Rossi, Asymptotic behavior of the best Sobolev trace constant in expanding and contracting domains, Comm. Pure Appl. Anal. 1(3) (2002), 359-378.

15. J. Fernández Bonder and J. D. Rossi, On the existence of extremals for the Sobolev trace embedding theorem with critical exponent, Bull. Lond. Math. Soc. 37(1) (2005), 119-125.

16. Y. Li and M. Zhu, Sharp Sobolev trace inequalities on Riemannian manifolds with boundaries. Comm. Pure Appl. Math. 50 (1997), 449-487.

17. J.-L. Lions, Some methods in the mathematical analysis of systems and their control (Kexue Chubanshe, Beijing, Gordon \& Breach, New York, 1981).

18. F. Murat and L. Tartar, H-convergence, in Topics in the Mathematical Modelling of Composite Materials (Cherkaev A. and Kohn R., Editors), Progress in Nonlinear Differential Equations and Their Applications, vol. 31 (Birkhäuser, Boston, 1997), 21-43.

19. G. Nguetseng, A general convergence result for a functional related to the theory of homogenization, SIAM J. Math. Anal. 20 (1989), 608-623.

20. M. W. Steklov, Sur les problèmes fondamentaux en physique mathématique, Ann. Sci. Ecole Norm. Sup. 19 (1902), 455-490. 